\title{
X-RAY OBERVATION OF THE NORMAL SPIRAL GALAXIES NGC2903 AND NGC628 WITH ASCA
}

T. MIZUNO, H. OHBAYASHI, N. IYOMOTO AND K. MAKISHIMA

Department of Physics, University of Tokyo

7-3-1 Hongo bunkyo-Ku, Tokyoo 113, Japan

\section{Introduction}

$\mathrm{X}$-ray emission from spiral galaxies without activity is thought to consist of low-mass X-ray binaries (LMXBs), and the Einstein observations established the relation between X-ray $(0.2-4 \mathrm{keV})$ and optical luminosities as $\log \left(\frac{L_{\mathrm{X}}}{L_{\mathrm{B}}}\right) \sim-4$ (Fabbiano, 1992). This relation has been used when discussing the activity other than LMXBs (Iyomoto, 1996). However, spectral information of Einstein Observatory was rather poor above $3 \mathrm{keV}$, where LMXBs would emit significant energy flux. Therefore we performed ASCA observations of two normal spirals, NGC2903 and NGC628, in order to better calibrate the $L_{\mathrm{X}}-L_{\mathrm{B}}$ relation.

\section{Spectral Analysis}

For both galaxies, the spectrum was extracted from a circular region centered on the source center and background spectrum was made from the source free region. The summary of the fit result are listed table 1 and 2 .

\section{Discussion}

We compared $L_{\mathrm{X}} / L_{\mathrm{B}}$ ratios of both garaxies with that of M31 (Makisnima, 1989). When comparing $X$-ray and optical luminosities, we calcurated $X$ ray luminosity of a hard component $\left(L_{\mathrm{X}}^{\text {hard }}\right)$ of energy band $2-10 \mathrm{keV}$, at this band LMXBs emit significant energy flux. Comparison are summarized in table 3. The $L_{\mathrm{X}} / L_{\mathrm{B}}$ ratios of M31 and NGC628 are similar to the value obtained by Einstein ( -4.4), but for NGC2903 ASCA exhibits smaller value than Einstein $(\sim-3.9)$ and the scattering of $L_{\mathrm{X}} / L_{\mathrm{B}}$ ratios of these three galaxies becomes smaller. This is owing to the fact that ASCA has sensitivity up to $10 \mathrm{keV}$, and separated the LMXBs emission from the soft component. About these three sources, $\log \left(\frac{L_{\mathrm{X}}}{L_{\mathrm{B}}}\right) \sim-4.4$. 


\section{References}

Fabbiano, G. et al. 1992 ApJS 80531

Makishima, K. et al. 1989 PASJ 41697

Iyomoto, N. et al. 1996 PASJ 48231

Tully, R.B. Nearby galaxies catalog (Cambridge Univ. Press)

\begin{tabular}{ccc}
\hline Parameter & $\begin{array}{c}\text { bremss }+ \\
\text { Raymond-Smith }\end{array}$ & $\begin{array}{c}\text { powerlaw }+ \\
\text { Raymond-Smith }\end{array}$ \\
\hline$k T(\mathrm{keV})$ of bremss or photon index & $8.2_{-2.0}^{+3.7}$ & $1.62_{-0.05}^{+0.07}$ \\
$k T(\mathrm{keV})$ of Raymond-Smith & $0.44_{-0.03}^{+0.05}$ & $0.47_{-0.04}^{+0.06}$ \\
abundance & $0.20 \pm 0.03$ & $0.28_{-0.04}^{+0.05}$ \\
\hline flux of hard component $\left(\mathrm{erg} / \mathrm{s} / \mathrm{cm}^{2}, 2-10 \mathrm{keV}\right)$ & $6.14 \times 10^{-13}$ & $6.86 \times 10^{-13}$ \\
\hline
\end{tabular}

TABLE 1 . best fit parameters and single-parameter $90 \%$ confidence limits of NGC2903.

\begin{tabular}{ccc}
\hline Parameter & bremss & powerlaw \\
\hline$k T(\mathrm{keV})$ or photon index & $12.1_{-6.1}^{+18.5}$ & $1.56_{-0.12}^{+0.15}$ \\
\hline flux $\left(\mathrm{erg} / \mathrm{s} / \mathrm{cm}^{2}, 2-10 \mathrm{keV}\right)$ & $2.43 \times 10^{-13}$ & $2.49 \times 10^{-13}$ \\
\hline
\end{tabular}

TABLE 2. best fit parameters and single-parameter $90 \%$ confidence limits of NGC628.

\begin{tabular}{cccc}
\hline & M31 & NGC2903 & NGC628 \\
\hline $\mathrm{L}_{X}(\mathrm{erg} / \mathrm{s}, 2-10 \mathrm{keV})$ & $3.9 \times 10^{39}$ & $2.9 \times 10^{39}$ & $2.7 \times 10^{39}$ \\
$\mathrm{~L}_{\mathrm{B}}(\mathrm{erg} / \mathrm{s})$ & $1.1 \times 10^{41}$ & $5.2 \times 10^{43}$ & $8.0 \times 10^{43}$ \\
$\log \left(\frac{\mathrm{L}_{\mathrm{X}}}{\mathrm{L}_{\mathrm{B}}}\right)$ & -4.5 & -4.3 & -4.5 \\
\hline
\end{tabular}

TABLE 3. comparison of X-ray and optical luminosities from three normal spiral galaxies. X-ray luminosity is calculated from the $2-10 \mathrm{keV}$ flux of thermal bremsstrahlung model. Optical luminosities are taken from Tully (1988). X-ray luminosity of M31 are calculated from the Ginga result (Makishima 1989). 